\title{
Transcriptome analysis reveals underlying immune response mechanism of fungal (Penicillium oxalicum) disease in Gastrodia elata Bl. f. glauca S. chow (Orchidaceae)
}

\author{
Yanhua Wang, Yugang Gao*, Pu Zang and Yue Xu
}

\begin{abstract}
Background: Gastrodia elata BI. f. glauca S. Chow is a medicinal plant. G. elata f. glauca is unavoidably infected by pathogens in their growth process. In previous work, we have successfully isolated and identified Penicillium oxalicum from fungal diseased tubers of G. elata f. glauca. As a widespread epidemic, this fungal disease seriously affected the yield and quality of G. elata f. glauca. We speculate that the healthy G. elata F. glauca might carry resistance genes, which can resist against fungal disease. In this study, healthy and fungal diseased mature tubers of $G$. elata $\mathrm{f}$. glauca from Changbai Mountain area were used as experimental materials to help us find potential resistance genes against the fungal disease.

Results: A total of 7540 differentially expressed Unigenes (DEGs) were identified (FDR $<0.01, \log 2 F C>2$ ). The current study screened 10 potential resistance genes. They were attached to transcription factors (TFs) in plant hormone signal transduction pathway and plant pathogen interaction pathway, including WRKY22, GH3, TIFY/JAZ, ERF1, WRKY33, TGA. In addition, four of these genes were closely related to jasmonic acid signaling pathway.

Conclusions: The immune response mechanism of fungal disease in G. elata f. glauca is a complex biological process, involving plant hormones such as ethylene, jasmonic acid, salicylic acid and disease-resistant transcription factors such as WRKY, TGA.
\end{abstract}

Keywords: Gastrodia elata Bl. f. glauca S. chow, Orchidaceae, Transcriptome, Fungal disease; immune response, Transcription factors, Changbai Mountain area

\section{Background}

Gastrodia elata Bl. f. glauca S. Chow is a form of Gastrodia elata Bl. (Orchidaceae). G. elata Bl., called tian $m a$ in Chinese, is a perennial monocotyledon. Its dry tuber is usually used as a precious traditional Chinese medicine Gastrodiae Rhizoma. The main active ingredients of Gastrodiae Rhizoma include gastrodin, phydroxybenzyl alcohol, parishin E, parishin B, parishin C and parishin [1]. It is recorded that Gastrodiae Rhizoma

\footnotetext{
*Correspondence: jInydxgyg@163.com

College of Chinese Medicinal Materials, Jilin Agricultural University, Changchun 130118, China
}

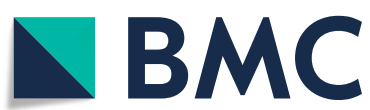

C C The Author(s). 2020 Open Access This article is licensed under a Creative Commons Attribution 4.0 International License, which permits use, sharing, adaptation, distribution and reproduction in any medium or format, as long as you give appropriate credit to the original author(s) and the source, provide a link to the Creative Commons licence, and indicate if changes were made. The images or other third party material in this article are included in the article's Creative Commons licence, unless indicated otherwise in a credit line to the material. If material is not included in the article's Creative Commons licence and your intended use is not permitted by statutory regulation or exceeds the permitted use, you will need to obtain permission directly from the copyright holder. To view a copy of this licence, visit http://creativecommons.org/licenses/by/4.0/ The Creative Commons Public Domain Dedication waiver (http://creativecommons.org/publicdomain/zero/1.0/) applies to the data made available in this article, unless otherwise stated in a credit line to the data. modic, calming liver and inhibiting yang, dispelling wind and relaxing chann and pharmacological research has shown that Gastrodiae Rhizoma has the effects of neuroregulation $[2,3]$, neuroprotection [4-7], improving memory [8, 9] and so on. It has auxiliary therapeutic effect on Alzheimer's disease (AD) $[8]$ and Parkinson's disease (PD) $[4,6,10,11]$ which are the common degenerative diseases nowadays.

Six G. elata varietas were described in Flora of Yunnan, and they are G. elata Bl. f. pilifera Tuyama, G. elata Bl. f. viridis Makino, G. elata Bl. f. glauca S. Chow, G. 
elata Bl. $f$. alba S. Chow, G. elata Bl. f. elata and G. elata Bl. f. flavida S. Chow. They were respectively called as Mao tian ma, Lv tian ma, Wu tian ma, Song tian ma, Hong tian ma, Huang tian $m a$ in Chinese. Among them, G. elata F. glauca is one of the most popular in the market because of its good shape and high dry rate. In China, G. elata Bl. f. glauca is mainly distributed in northeastern Yunnan, western Guizhou, southern Sichuan and Changbai Mountain area. G. elata Bl. f. glauca is not only a traditional Chinese medicinal material in Changbai Mountain, but also one of the most vital special economic crops in Jilin Province. However, the genetic research of G. elata Bl. f. glauca in Changbai Mountain area is almost blank.

G. elata $\mathrm{Bl}$. is an obligate fungal heterotrophic plant with highly degraded leaves and bracts. More than $80 \%$ of its life cycle exists underground in the form of tuber, depending almost entirely on fungi to provide nutrient [12]. It is closely related to at least two types of fungi: Mycena to promote seed germination and Armillaria Mellea to ensure reproductive growth. The growth and development of G. elata Bl.usually goes through seed, protocorm, juvenile tuber (also called $M i m a$ in Chinese), immature tuber (also called Bai ma in Chinese), mature tuber (also called Jian $m a$ in Chinese), scape, flower, and fruit [12]. During the growth and development of G. elata, it is susceptible to infection by non-essential fungi such as Penicillium [13], Ilyonectria robusta [14] and Trichoderma hamatum [15]. The main natural diseases that occur on G. elata Bl. f. glauca are soft rot, black spot and mildew. In our previous studies, two fungal pathogens (Penicillium oxalicum, Candida vartiovaarae) were isolated and identified from diseased G. elata Bl. f. glauca. Fungal disease induced by Penicillium oxalicum had widespread prevalence in Changbai Mountain area [13]. Diseased G. elata Bl. tubers become moldy, soft and rotted [13]. Fungal disease incidence in G. elata Bl. f. glauca is $6 \% \sim 17 \%$, giving rise to a $10 \% \sim 30 \%$ reduction in yield [16]. So far, there is no research report on disease resistance breeding of G. elata Bl. f. glauca by means of genomics tools. Therefore, it is imperative to carry out research on immune response mechanism of fungal disease in $G$. elata Bl. $f$. glauca.

Obviously, under the same condition of being infected, physiologically healthy G. elata Bl. f. glauca probably have potential disease resistance genes. We intended to screen candidate genes for disease resistance through differential expression analysis. In this study, a detailed comparison was made between healthy and fungal diseased G. elata Bl. f. glauca tubers by means of transcriptome sequencing and bioinformatics analysis. It may provide a new insight for the breeding of disease resistant varieties of G. elata Bl. f. glauca.

\section{Results}

Sequencing overview

$7.89 \times 10^{10}$ base (healthy group) and $6.45 \times 10^{10}$ base (fungal diseased group) clean data were generated by sequencing platform. GC content ranged from 47.16 to $49.09 \%$, and Q30 of each sample was above $92.92 \%$ (Additional file: Table S1). It was showed that sequencing fragments had high randomness and reliability (Additional file: Figure S1A). After transcript de novo assembly, 60,324 Unigenes in total were obtained, and the N50 was $2409 \mathrm{~kb}$. Furthermore, 19,670 (32.61\%) of them were over $1 \mathrm{~kb}$ in length (Additional file: Figure S1B). All these indicative data displayed high assembly integrity.

\section{Functional annotation and differential expression analysis DEGs annotation and function classification}

The most DEGs annotated into $\mathrm{nr}$ (RefSeq nonredundant proteins), while the least annotated into KEGG (Fig. 1a). The venn diagram displayed the set of DEGs in four common databases which covered nearly all annotated DEGs (Fig. 1b). It was learned that DEGs between healthy and fungal diseased samples chiefly classified into "signal transduction mechanisms", "carbohydrate transport and metabolism", "defense mechanisms", "energy production and conversion", "general function prediction only", "post-translation modification, protein turnover, chaperones", "translation, ribosomal structure and biogenesis" (Fig. 1c, d).

\section{GO enrichment and KEGG enrichment analysis}

2482 DEGs were enriched into 3958 GO terms. GO terms are usually classified into 3 categories: biological process (BP), cellular component (CC), molecular function (MF). Here, 2363 (59.70\%) of these GO terms attached to BP, 509 (1.49\%) belonged to CC, and 1086 (27.44\%) were part of MF. 36 GO terms involved signal transduction, and $24 \mathrm{GO}$ terms involved phytohormone. By Kolmogorov-Smirnov test, $421 \mathrm{GO}$ terms were significantly enriched $(p<0.05)$. Part of them were showed in Additional file: Table S2 $(p<0.05)$ and top 30 were displayed as Fig. 2a.

122 pathways (Additional file: Table S3) were enriched and top 50 was showed as Fig. 2c. The enrichment degree was based on $p$ value and enrichment factor (Fig. 2b). Nine pathways were significantly enriched $(p<$ 0.05 ), and they attached to three pathway categories: metabolism, environmental information processing, organismal systems (Table 1).

\section{Differential expression analysis}

A total of 7540 DEGs were identified. 4326 of these DEGs were up-regulated in diseased group, and 3214 were down-regulated (Fig. 3a, b). In addition, 40,440 Unigenes did not demonstrate significantly differential 


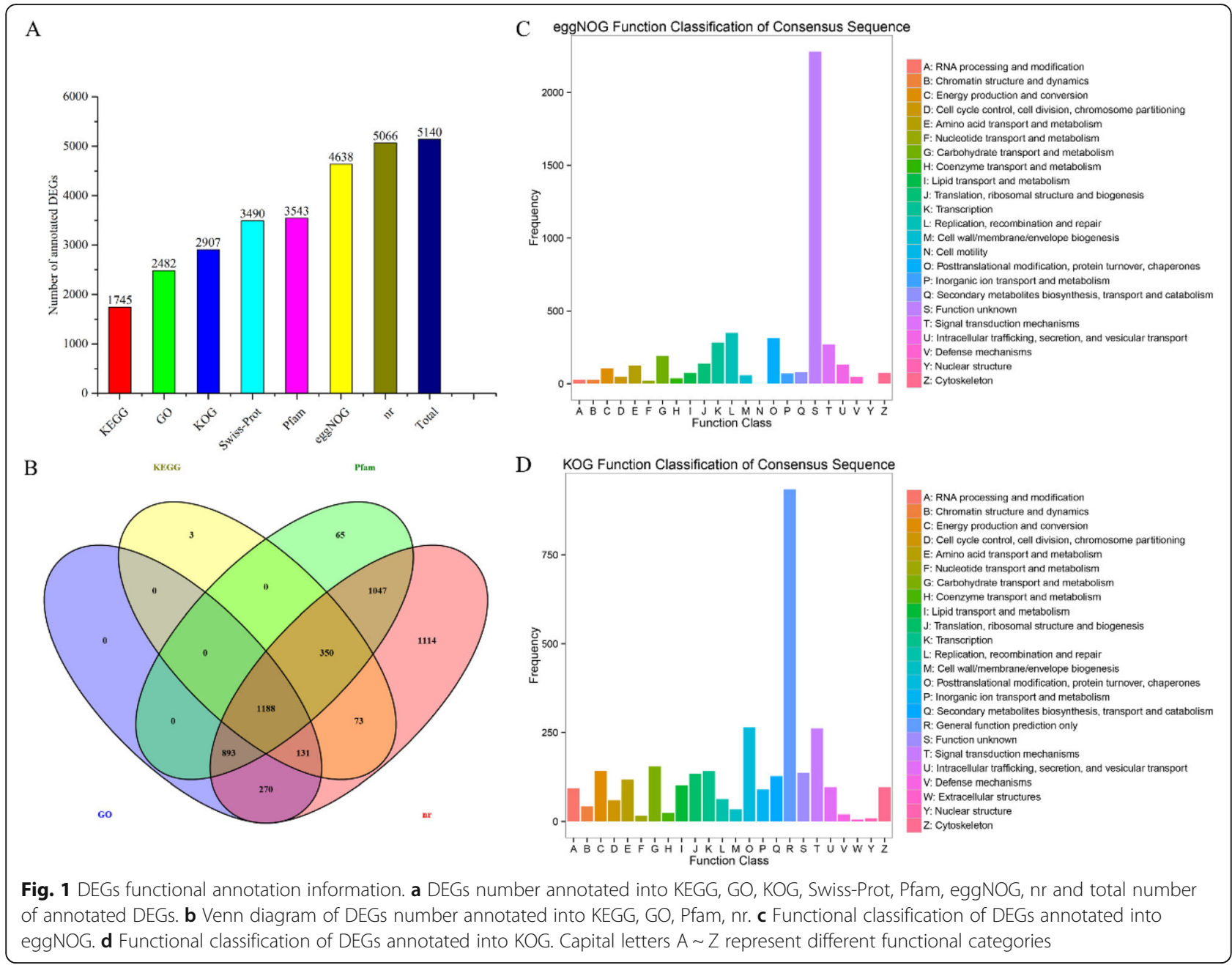

expression. Overall, DEGs between healthy and diseased samples accounted for $15.71 \%$ of all Unigenes.

\section{Transcription factor prediction}

By the standard of FDR $<0.01$ and FC $>2,1295$ DEGs were identified as transcription factors with transcription factor prediction tool (Fig. 4). Here, transcription factor family covers transcription factor (TF), transcription regulator (TR), protein kinases (PK). It could be clear to see that many DEGs were the members of transcription factor families MYB, ERF, C2H2, NAC, bHLH, C3H, WRKY, bZIP, GRAS, PHD, SNF2, SET.

\section{KEGG pathways analysis}

The current study paid close attention to pathways related to plant immune response. In plant-pathogen interaction map, only one node displayed negative regulation, and other 14 nodes revealed positive regulation (Fig. 5). In plant hormone signal transduction map, 6 nodes were upregulated, 10 nodes were down-regulated, and 6 were mix-regulated (Fig. 6). In brassinosteroid biosynthesis map, 2 nodes showed positive regulation, 3 nodes displayed negative regulation, and 2 nodes covered both upregulated genes and down-regulated genes (Fig. 7).

\section{Candidate genes responding to fungal disease in G. elata Bl. f. glauca}

Comprehensively considering gene expression levels (FPKM $>10)$, significance of differential expression $(\mathrm{FDR}<0.01,|\log 2 \mathrm{FC}|>2)$ and literature related to plant immune response [17-21], 10 candidate genes responding to fungal disease in G. elata Bl.f. glauca were found (Fig. 8; Table 2).

\section{Real-time quantitative polymerase chain reaction (qRT- PCR) analysis}

Seven genes showed higher expression in the fungal diseased group $(p<0.05)$, and one displayed negative expression $(p<0.05)$. Only the gene labeled as c32310 revealed no significant difference in relative expression level between the two groups $(p>0.05)$. In addition, there was no 


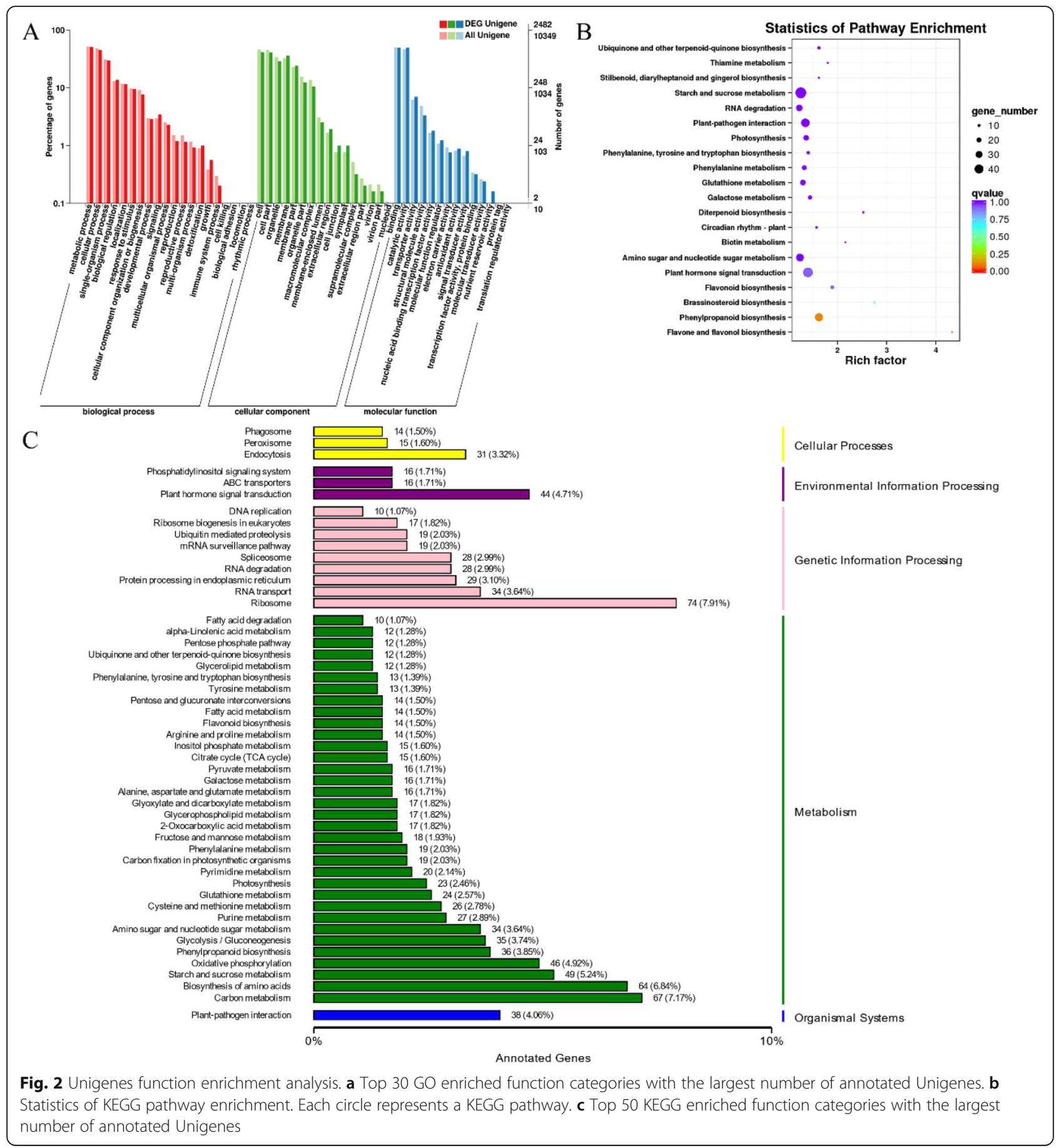

quantitative result for one gene, which may be due to unreasonable primer design.

\section{Discussion}

\section{Pathways related to plant immune response}

So far, it has been proved that plant immune response is relative to plant-pathogen interaction, plant hormone signal transduction, and pathways about certain secondary metabolite biosynthesis or metabolism [22-26]. Consistently, we got similar results in this study (Table 1).

In plant-pathogen interaction pathway, all except WRKY1/2 were up-regulated. They were CDPK (calcium-dependent protein kinase), Rboh (respiratory burst oxidase homolog), CNGC (cyclic nucleotide gated channel), calcium-binding protein CML (calmodulin-like protein), LRR (leucine-rich repeat) receptor-like serine/ threonine-protein kinase FLS2, MEKK1 (mitogen- 
Table 1 KEGG pathway enrichment analysis $(p<0.05)$

\begin{tabular}{|c|c|c|c|c|c|c|}
\hline Pathway category & Pathway description & Specific pathway & ko ID & DEG & $\begin{array}{l}\text { All } \\
\text { Unigene }\end{array}$ & $p$ \\
\hline \multirow[t]{7}{*}{ Metabolism } & Carbohydrate metabolism & Starch and sucrose metabolism & ko00500 & 49 & 169 & 0.041 \\
\hline & $\begin{array}{l}\text { Metabolism of cofactors and } \\
\text { vitamins }\end{array}$ & $\begin{array}{l}\text { Ubiquinone and other terpenoid-quinone } \\
\text { biosynthesis }\end{array}$ & ko00130 & 12 & 32 & 0.047 \\
\hline & \multirow{2}{*}{$\begin{array}{l}\text { Metabolism of terpenoids and } \\
\text { polyketides }\end{array}$} & Brassinosteroid biosynthesis & ko00905 & 7 & 11 & 0.005 \\
\hline & & Diterpenoid biosynthesis & ko00904 & 7 & 12 & 0.009 \\
\hline & \multirow{3}{*}{$\begin{array}{l}\text { Biosynthesis of other secondary } \\
\text { metabolites }\end{array}$} & Flavone and flavonol biosynthesis & ko00944 & 5 & 5 & 0.001 \\
\hline & & Phenylpropanoid biosynthesis & ko00940 & 36 & 96 & 0.001 \\
\hline & & Flavonoid biosynthesis & ko00941 & 14 & 32 & 0.008 \\
\hline $\begin{array}{l}\text { Environmental Information } \\
\text { Processing }\end{array}$ & Signal transduction & Plant hormone signal transduction & ko04075 & 44 & 136 & 0.008 \\
\hline Organismal Systems & Environmental adaptation & Plant-pathogen interaction & ko04626 & 38 & 122 & 0.023 \\
\hline
\end{tabular}

activated protein kinase kinase kinase 1), MKK4/5 (mitogen-activated protein kinase kinase 4/5), WRKY transcription factor 33, WRKY transcription factor 22, RIN4 (RPM1-interacting protein 4), serine/threonine-protein kinase PBS 1, molecular chaperone HtpG. Biological processes these up-regulated genes principally involved were hypersensitive response (HR), cell wall reinforcement, defense-related gene induction, phytoalexin accumulation and miRNA production. Some of these genes were involved in PAMP-triggered immunity. Only WRKY transcription factor 2 displayed down-regulated expression, and it was connected with HR, defense-related gene induction and programmed cell death.

In plant hormone signal transduction, we learned that GH3 (auxin responsive glycoside hydrolase 3 gene family), AHP (histidine-containing phosphotransfer protein), ARR-B (two-component response regulator ARR-B family), PIF4 (phytochrome-interacting factor 4), ERF1 (ethylene-responsive transcription factor 1), JAZ (jasmonate ZIM domain-containing protein) were upregulated. AUX1 (auxin influx carrier), ARF (auxin response factor), CRE1 (cytokinin receptor enzyme),
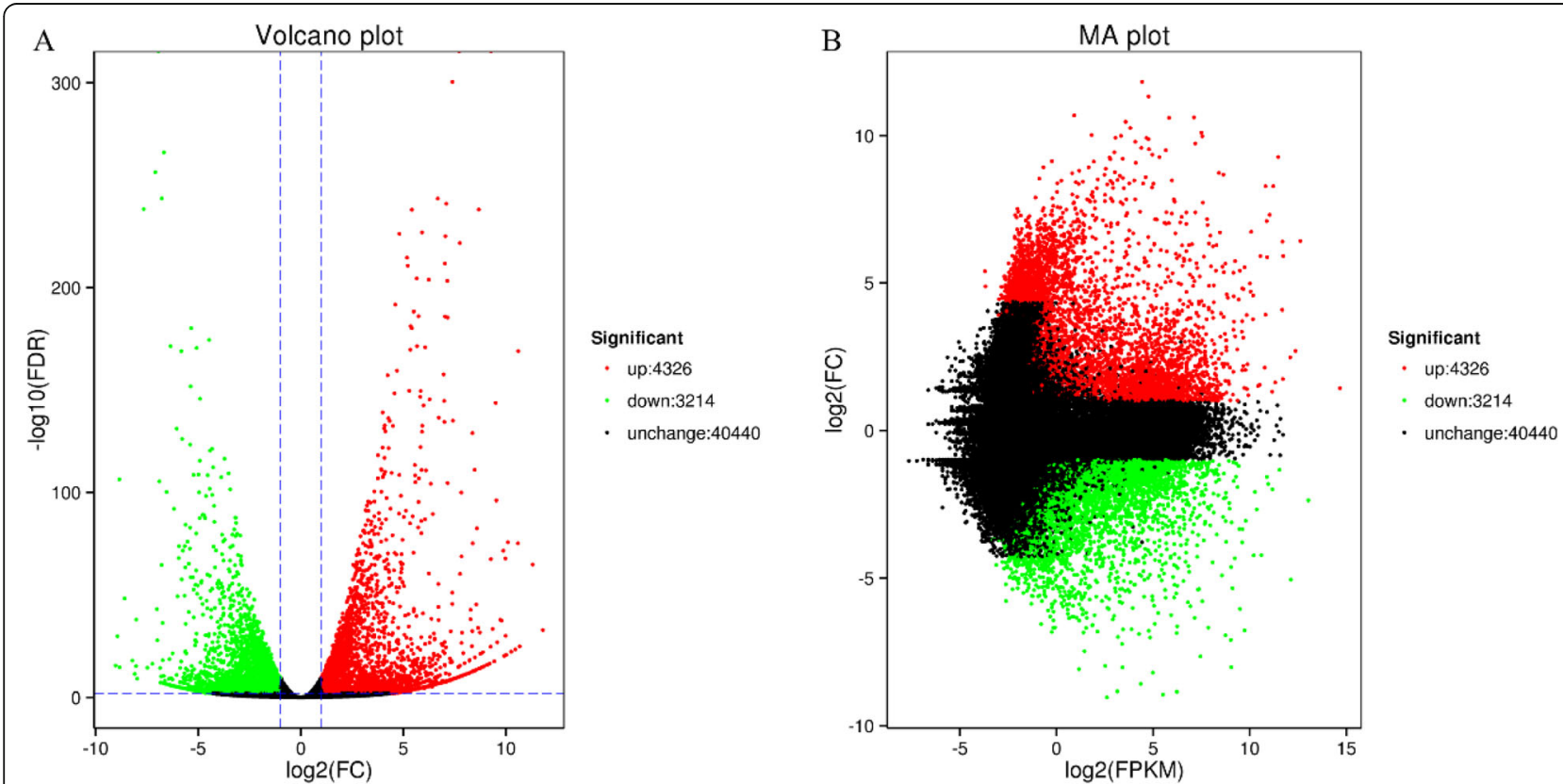

Fig. 3 Differential expression analysis. Each dot represents a gene. Green represents down-regulation; red represents up-regulation; black represents non-differentially expression. a Volcano map of DEGs. X-axis represents the log2(FC) value. The greater the absolute value of log2(FC), the greater the difference of gene expression level between the two groups. Y-axis represents the negative log 10(FDR) value. The larger the value, the more significant the difference, as well the more reliable the DEGs. b MA plot of DEGs. MA plot displays the normalized gene distribution. $X$-axis represents the $\log 2(F P K M)$ value, and Y-axis represents the $\log 2(\mathrm{FC})$ value 


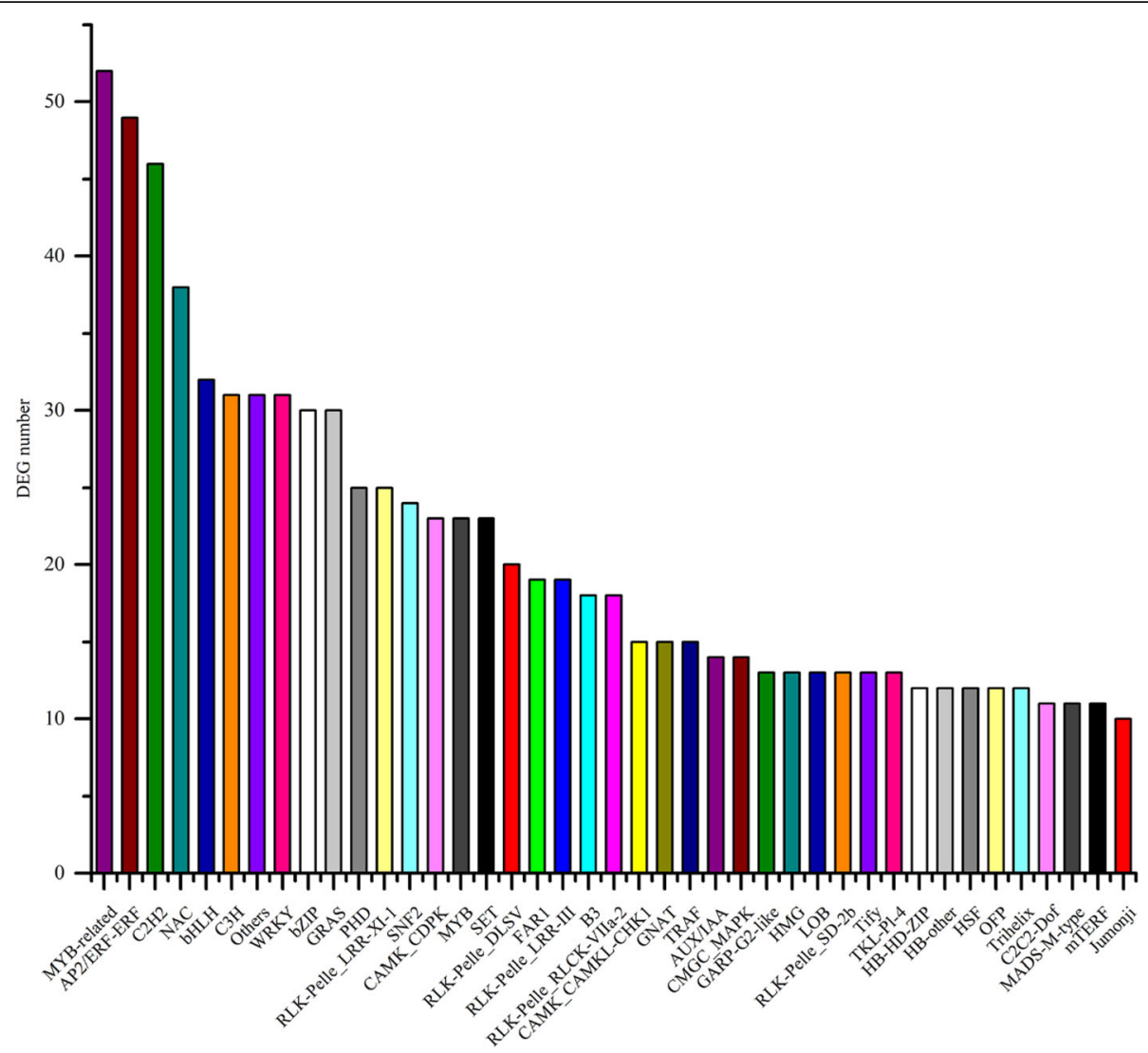

Fig. 4 Transcription factor prediction. X-axis represents the names of transcription factor family, and Y-axis represents the number of DEGs

DELLA protein, PP2C (protein phosphatase 2C), EIN2 (ethylene-insensitive protein 2), BZR1/2 (brassinosteroid resistant 1/2), JAR1 (jasmonic acid-amino synthetase), COI1 (coronatine-insensitive protein 1), transcription factor TGA showed down-regulated. As it described, transcription factor TGA is connected with disease resistance [27]. DEGs in this pathway involved many biological processes, such as cell enlargement, plant growth, cell division, shoot initiation, stem growth, stomatal closure, seed dormancy, fruit ripening, senescence, monoterpenoid biosynthesis, indole alkaloid biosynthesis, cell elongation, of course, disease resistance as well (Fig. 6). Above biological processes usually accompanied by phosphorylation $(+\mathrm{p})$, dephosphorylation $(-\mathrm{p})$, ubiquitination $(+\mathrm{u})$. Phosphorylation and ubiquitination are common post-translational modification of proteins. They play an important role in pattern-triggered immunity (PTI), and simultaneously be necessary to receptor complex activation signals and cell homeostasis [28]. Phytohormone played a vital role in this pathway. They included jasmonic acid (JA), salicylic acid (SA), ethylene (ET), brassinosteroid (BR), auxin, cytokinine, gibberellin, abscisic acid.

In fact, plant hormones do play a vital role in the process of plant-pathogen interaction. The current study found a large number of DEGs annotated to signal transduction mechanisms by means of functional annotation. Furthermore, lots of DEGs were markedly enriched into plant hormone signal transduction pathway. Consistently, it has been reported that auxin $[29,30]$, cytokinins [31, 32], ethylene [30, 33-35], gibberellin [36], abscisic acid [30, 37, 38], brassinosteroids [35], salicylic acid [30, 33, 39], jasmonic acid [30, 33, 39-41], strigolactones [42] can actively participate in disease response. Among them, salicylic acid signal transduction and jasmonic acid/ethylene signal transduction are considered as the most common plant hormone signal transduction pathways in response to biological or abiotic stress. It could even be said that the plant resistance against pathogen is initially stimulated by gene expression regulated by transcription factors and ultimately be mediated by plant hormones. Therefore, if possible, it is necessary to study phytohormone metabolism of G. elata $\mathrm{Bl}$. $f$. glauca in the following work.

Brassinosteroid is one of crucial phytohormone closely related to plant growth and stress response. In brassinosteroid biosynthesis pathway, CYP90D2 (steroid 3oxidase) showed up-regulated expression; CYP90A1 (cytochrome P450 family 90 subfamily A polypeptide 1) displayed down-regulated expression; CYP734A1/BAS1 (PHYB activation tagged suppressor 1) was mix- 
PLANT-PATHOGEN INTERACTION

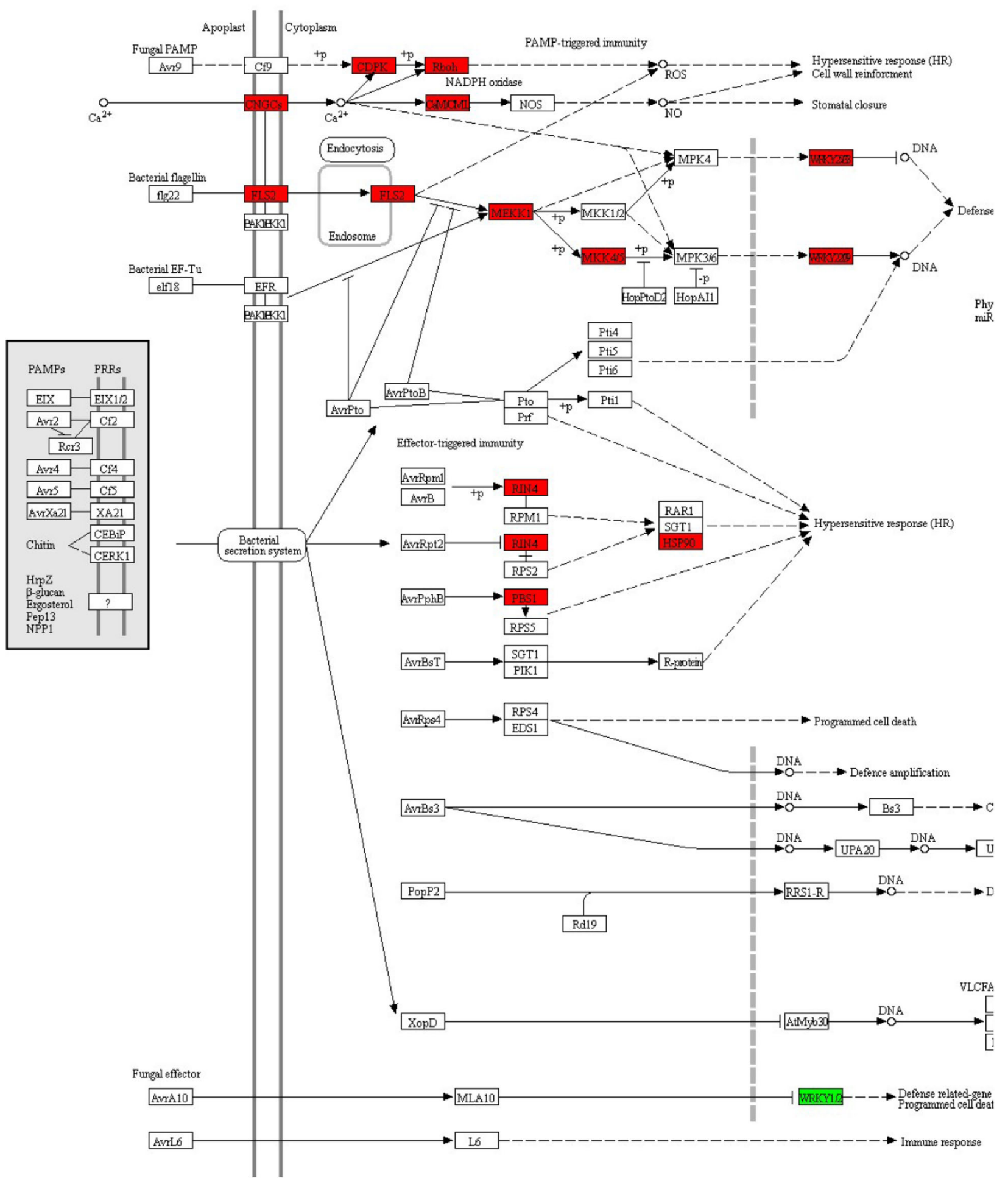

Fig. 5 Plant-pathogen interaction map. Positive regulation is highlighted in red; negative regulation is highlighted in green 
PLANT HORMONE SIGNAL TRANSDUCTION
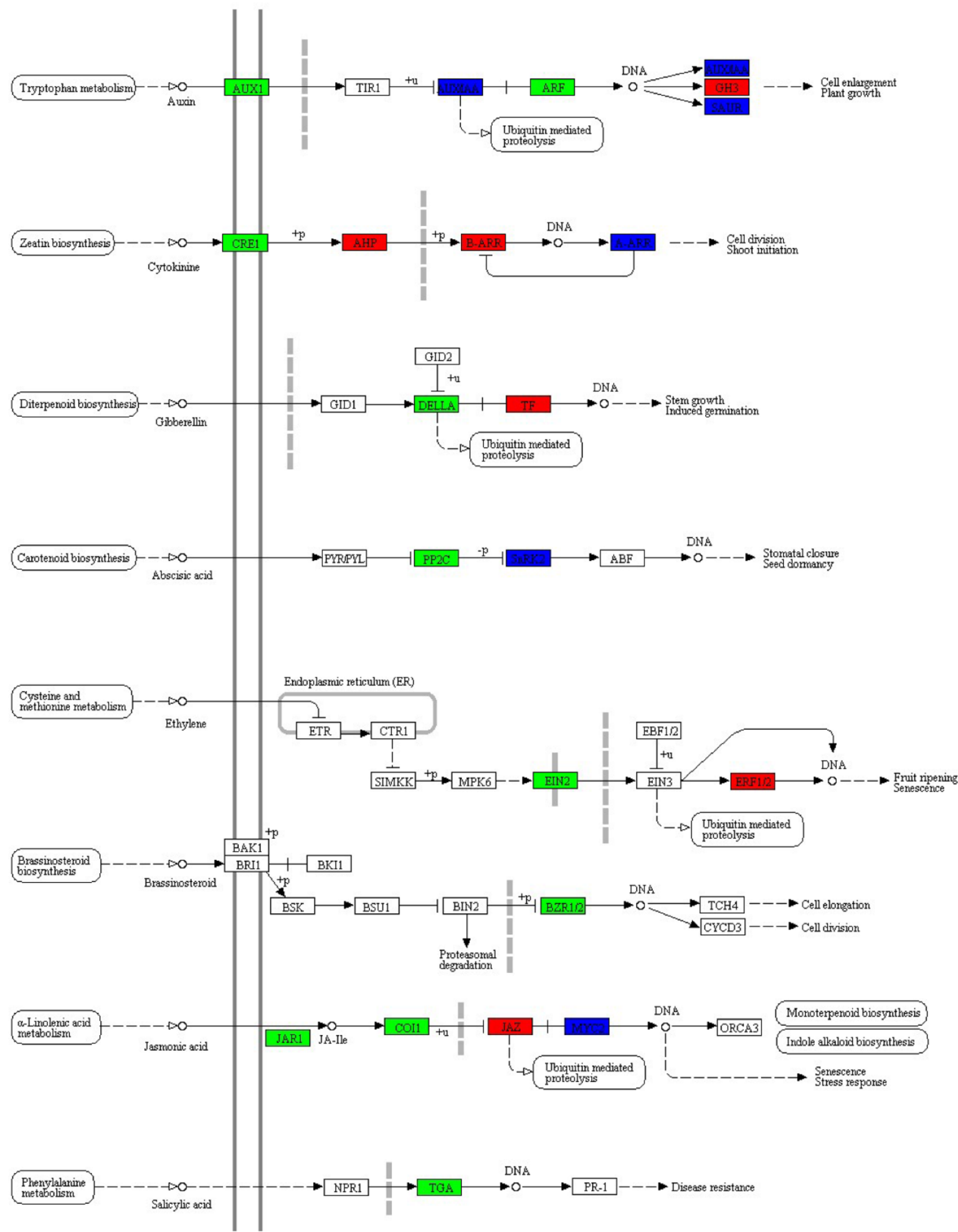

Fig. 6 Plant hormone signal transduction map. Positive regulation is highlighted in red; negative regulation is highlighted in green; mixed regulation is highlighted in blue 


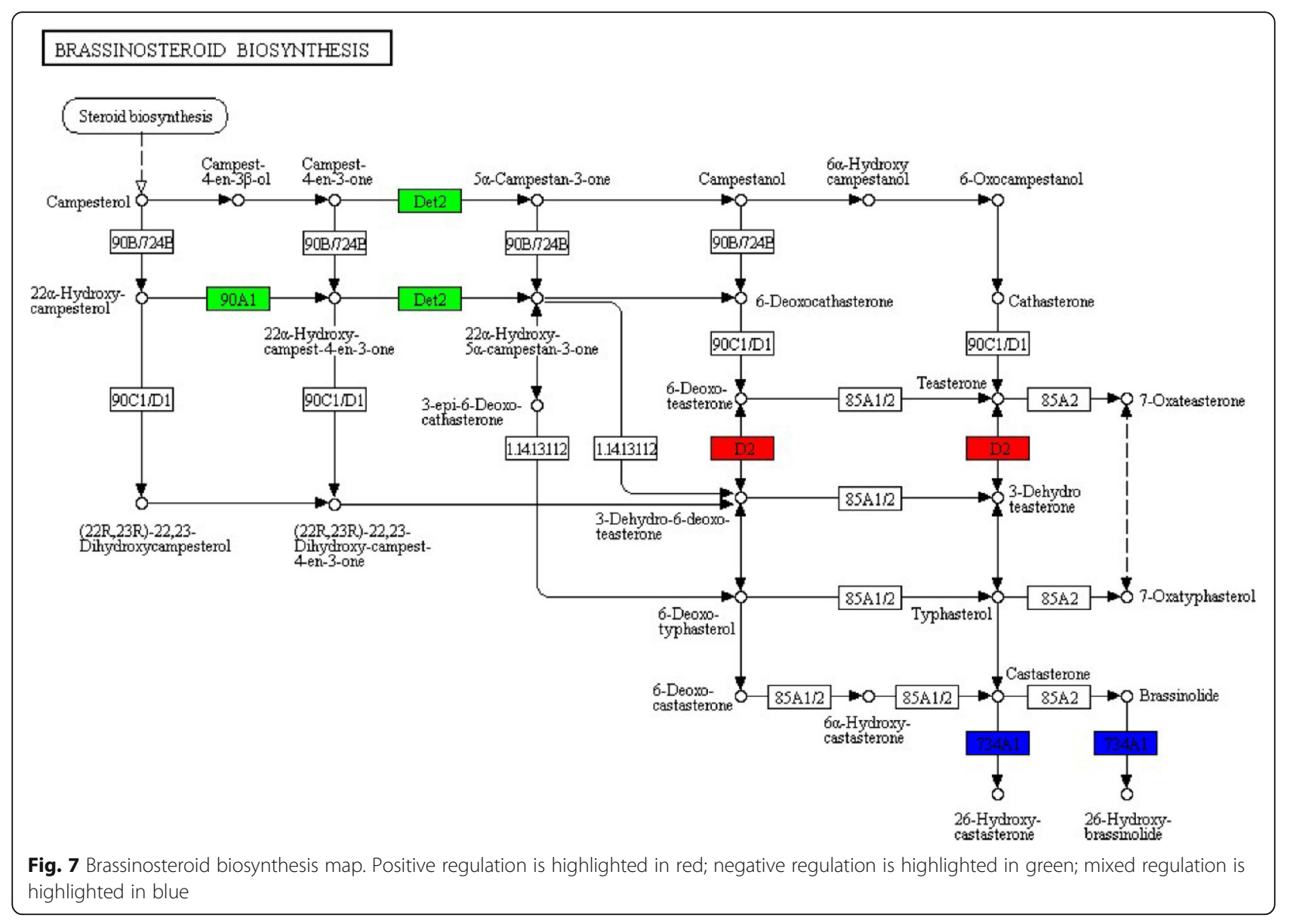

regulated, with two genes up-regulated and one gene down-regulated (Fig. 7).

The current study also found numerous DEGs appear in the pathways of secondary metabolites biosynthesis. CYP75B1 and CYP75A showed significant differential expression in flavone and flavonol biosynthesis pathway. 4CL, CYP84A appeared in phenylpropanoid biosynthesis pathway. 4CL and CYP73A displayed positive regulation in ubiquinone and other terpenoid-quinone biosynthesis pathway. $4 \mathrm{CL}$ is a key enzyme in the synthesis of lignin and it can response to osmotic stress by regulating secondary cell wall development and stomatal [43]. This may be a part of fungal disease immune response mechanism in G. elata Bl. f. glauca.

In starch and sucrose metabolism pathway, DEGs involved in fructose and glucose synthesis were mainly positively regulated, and they were fructokinase (EC: 2.7.1.4), beta-fructofuranosidase (EC:3.2.1.26), hexokinase (EC:2.7.1.1), phosphoglucomutase (EC:5.4.2.2) and UTP-glucose-1-phosphate uridylyltransferase (EC: 2.7.7.9); while several DEGs involved in starch and glycogen synthesis mainly showed negative regulation, and they covered 1,4-alpha-glucan branching enzyme (EC:2.4.1.18), starch synthase (EC:2.4.1.21), 4-alphaglucanotransferase (EC:2.4.1.25) and so on.
In summary, fungal disease immune response is a complex process involving multiple biological processes. It covers more than one gene and one gene does not work in single pathway. That is to say, one gene may perform more than one function simultaneously. These significantly enriched pathways might well reveal the underlying immune response mechanism of fungal disease in G. elata Bl. f. glauca.

\section{Defense-related transcription factors}

It has been proved that many a transcription factor could directly or indirectly regulate plants immune response [26, 44-62]. Here, the current study got the similar result (Fig. 4). Exceptionally, according to transcription factor prediction, some $\mathrm{C} 3 \mathrm{H}$ genes were differentially expressed in two groups. However, present reports about $\mathrm{C} 3 \mathrm{H}$ are mainly related to cold resistance, rather than disease resistance $[63,64]$.

\section{Resistance genes ( $R$ genes)}

Resistance genes ( $R$ genes) were classified into nine types based on intracellular and extracellular pathogen recognition mechanisms [65]. Here, the current study discovered potential R genes in G. elata Bl. f. glauca were 


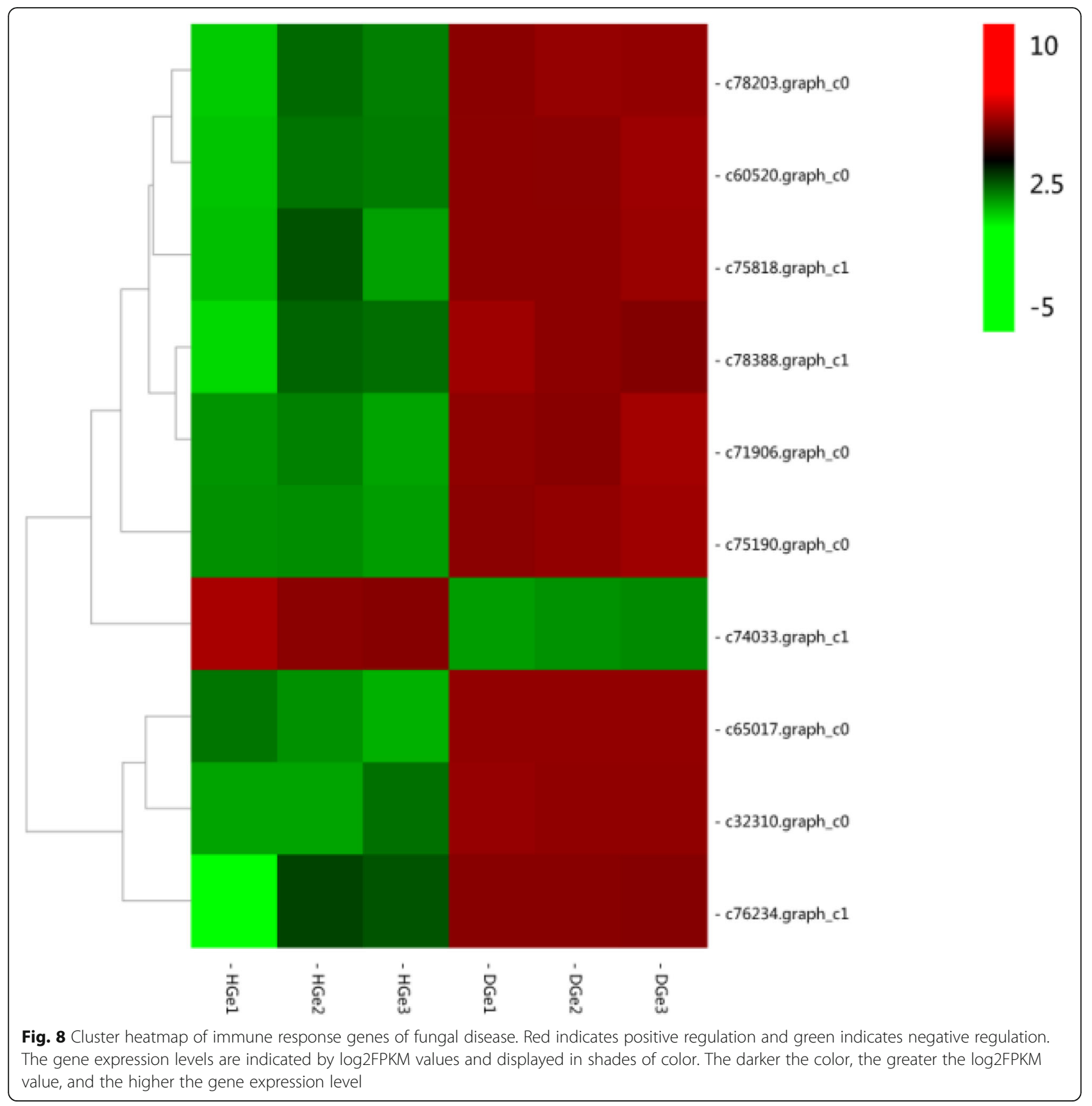

probably the member of transcription factor families like WRKY, GH3, TIFY/JAZ, CML, ERF, TGA. Coincidently, it has been reported that above transcription factors do be widely involved in various defense responses $[26,66-78]$. It is reported that GH3 and CML can also regulate fruit development $[79,80]$. To verify the accuracy of transcriptome sequencing, qRT-PCR test was performed, and the results were basically consistent with transcriptome sequencing (Fig. 9). However, it still needs further study on how these genes perform their functions in respond to fungal disease in G. elata Bl. f. glauca.

\section{Potential immune response mechanism of fungal disease} in G. elata BI. f. glauca

Plant immune response mechanisms mainly include PAMP-triggered immunity (PTI), effector-triggered immunity (ETI) and systemic acquired resistance (SAR). ETI is usually accompanied by the occurrence of hypersensitivity reaction (HR), giving rise to programmed cell death (PCD). Moreover, ETI can induce SAR. As is known to all, PTI and SAR are nonspecific immunity, while ETI is specific immunity [81]. From the current study, the immune response mechanism of fungal disease in G. elata Bl. f. glauca 
Table 2 Information of disease resistance genes. ko04626: plant-pathogen interaction; ko04075: plant hormone signal transduction. K13425: WRKY22; K14487: GH3; K13464: JAZ; K13448: CML; K14516: ERF1; K13424: WRKY33; K14431: TGA

\begin{tabular}{|c|c|c|c|c|c|c|}
\hline Gene ID & FDR & Log2FC & Regulated & pathway & $\begin{array}{l}\text { KEGG } \\
\text { entry }\end{array}$ & nr annotation \\
\hline c65017.graph_c0 & $8.65 \mathrm{E}-170$ & 10.60551 & up & ko04626 & K13425 & probable WRKY transcription factor 27, partial [Phalaenopsis equestris] \\
\hline c32310.graph_c0 & 7.67E-97 & 9.531941 & up & ko04075 & K14487 & $\begin{array}{l}\text { probable indole-3-acetic acid-amido synthetase GH3.1 [Phalaenopsis } \\
\text { equestris] }\end{array}$ \\
\hline C75818.graph_c1 & $5.48 \mathrm{E}-19$ & 5.547811 & up & ko04075 & K13464 & protein TIFY 10a-like [Dendrobium catenatum] \\
\hline C76234.graph_c1 & $1.23 \mathrm{E}-15$ & 5.462372 & up & ko04075 & K13464 & protein TIFY 10c-like [Dendrobium catenatum] \\
\hline c60520.graph_c0 & $1.11 \mathrm{E}-77$ & 4.956140 & up & ko04075 & K13464 & protein TIFY 10a-like [Dendrobium catenatum] \\
\hline c75190.graph_c0 & $3.26 \mathrm{E}-110$ & 4.430928 & up & ko04626 & K13448 & probable calcium-binding protein CML18 [Phalaenopsis equestris] \\
\hline c78203.graph_c0 & 7.49E-63 & 4.276427 & up & ko04075 & K14516 & ethylene-responsive transcription factor 1B-like [Dendrobium catenatum] \\
\hline c78388.graph_c1 & $1.21 \mathrm{E}-46$ & 3.499401 & up & ko04075 & K13464 & protein TIFY 10a-like [Dendrobium catenatum] \\
\hline c71906.graph_c0 & $6.55 E-51$ & 2.652510 & up & ko04626 & K13424 & $\begin{array}{l}\text { WRKY transcription factor WRKY24-like isoform X1 [Dendrobium } \\
\text { catenatum] }\end{array}$ \\
\hline c74033.graph_c1 & $8.03 \mathrm{E}-27$ & -2.15672 & down & ko04075 & K14431 & transcription factor TGA1-like [Dendrobium catenatum] \\
\hline
\end{tabular}

involves all above three kinds of mechanisms in the whole process of infection.

In this study, many genes related to stress response and disease resistance demonstrated high expression and significant difference. They were members of certain transcription factor families, like WRKY, GH3, JAZ, CML, ERF, TGA. Furthermore, these genes were closely connected with derivatives of jasmonic acid, salicylic acid, brassinosteroid, ethylene and auxin. By BLAST (https://blast.ncbi.nlm.nih.gov/Blast.cgi), it is revealed that amino acid sequences of four JAZ genes in G. elata family were highly similar to certain gene sequences in Dendrobium catenatum, Phalaenopsis equestris, Apostasia shenzhenica (Fig. 10). They were all belong to TIFY10 family.

\section{Conclusions}

In conclusion, immune response mechanism of fungal disease in G. elata Bl. f. glauca is quite complicated. JA/ ET signal transduction and SA signal transduction show positive regulation in this progress. Firstly, the expression of JAZ and ERF1 positively induces ubiquitin mediated proteolysis. Secondly, the expression of TGA indirectly triggered disease resistance in physiologically healthy group, rather than in diseased group. Thirdly, brassinosteroid biosynthesis also makes contributions to fungal disease response. CYP90A1 and CYP90D2 display down-regulation and up-regulation, respectively. Last but not least, auxin signaling pathway involves in fungal disease response actively. However, JA/ET signaling pathway is undoubtedly the most highlighted. As the candidate genes response to fungal disease in G. elata Bl. f. glauca, their specific functions still need to be further verified. Of course, more insight into the molecular mechanisms of fungal disease response also requires to be revealed. If possible, we intend to perform transgenic functional verification of these candidate genes.

\section{Methods}

Plant material and growth conditions

Healthy tubers (HGe, Accession ID: SAMN14380862) and fungal diseased tubers (DGe, Accession ID: SAMN14380861) were used in this experiment [13]. These experimental materials were all from the Changbai Mountain area. They were identified as mature tubers of G. elata Bl. f. glauca S. Chow in Jilin Agricultural University.

Fresh tubers were collected in October, 2018 from a planting base $\left(126^{\circ} 44^{\prime} 20^{\prime \prime} \mathrm{E}, 42^{\circ} 24^{\prime} 30^{\prime \prime} \mathrm{N}\right)$ attached to JINGZHEN TIANMA Co., Ltd. It is located in Jingyu County, Baishan City, Jilin Province, PR China. The manager of this company gave permission for sampling. Jingyu County is located in the western foot of Changbai Mountain and the upper reaches of Songhua River, PR China, with average altitude $775 \mathrm{~m}$, annual average temperature $2.5^{\circ} \mathrm{C}$, effective accumulated temperature $2224^{\circ} \mathrm{C}$, annual average rainfall $767.3 \mathrm{~mm}$, frost-free period $110 \mathrm{~d}$ or so. According to data from China Meteorological Administration (http://data.cma.cn/data/ weatherBk.html), the monthly mean temperature range from $-17^{\circ} \mathrm{C}$ to $21^{\circ} \mathrm{C}$, the monthly relative humidity ranged from 58 to $83 \%$, and the monthly rainfall ranged from 7.8 to $207.4 \%$ (Additional file: Figure. S2). The vegetative growth of G. elata Bl. f. glauca is usually from April of the first year to October of the following year or even longer. However, it usually takes only from April to June for it to complete the reproductive growth process. And it takes about $1.5 \sim 3$ years to mature for harvest the tuber [82]. The soil type of local area where they grow is dark brown soils on hillside. 
c65017

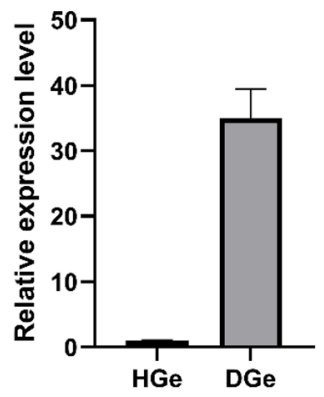

c76234

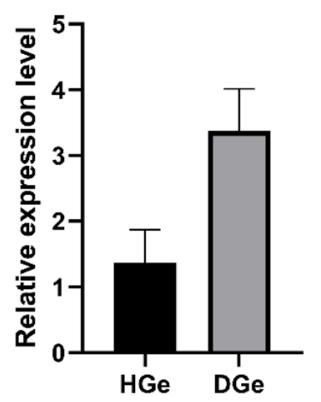

c78388

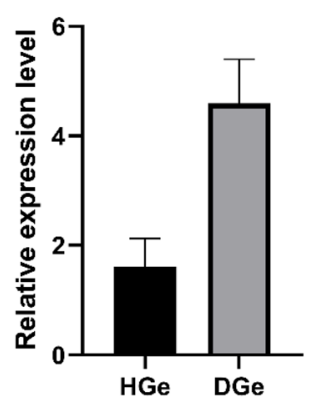

c32310

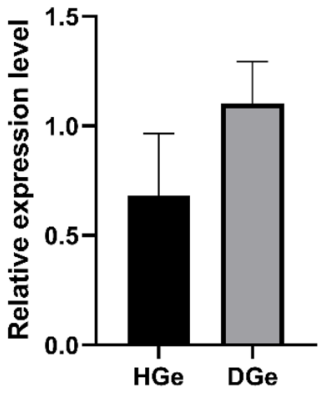

c60520

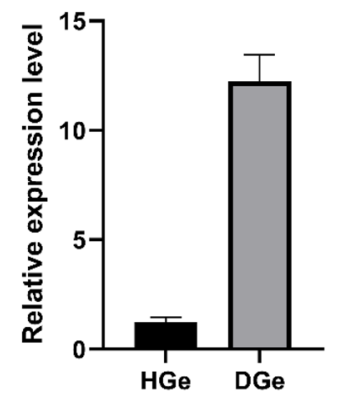

c71906

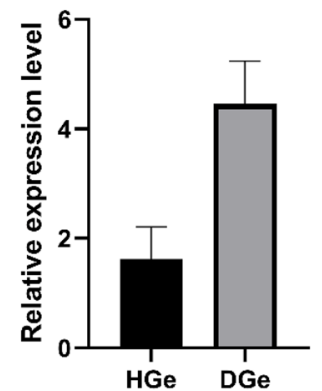

c75818

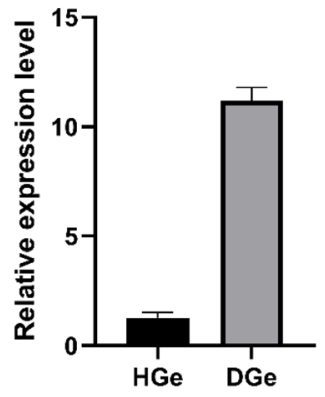

c75190

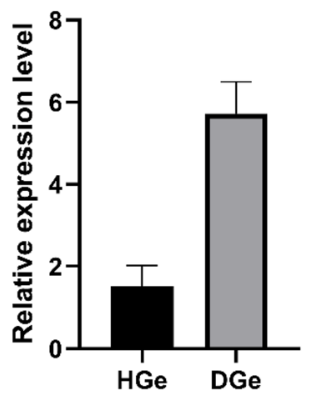

c74033

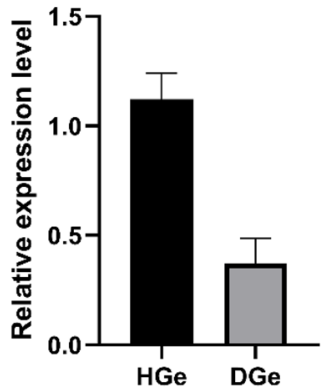

Fig. 9 Relative expression levels of nine potential immune response genes by qRT-PCR assays. The relative expression levels are displayed with the $2^{-\Delta \Delta C t}$ values. All genes but c32310 show significant differential expression between HGe and DGe groups $(p<0.05)$

\section{RNA extraction}

Fresh G. elata Bl.f. glauca tubers used for RNA extraction were washed with sterile water, and after surface disinfection, $100 \mathrm{mg}$ or so healthy tissue was cut near the infected tissue from diseased tubers. Tissues were taken from the same part of healthy tubers to keep uniformity between the two samples, each of which has three biological replications. The total RNA was extracted from each tissue using RNAprep Pure Plant Total RNA Extraction Kit (Polysaccharides \& Polyphenolics-rich) (centrifugal column type, catalog No. DP441) and referring to the manual on its official website (https://www.tiangen.com/). RNA was quantified in an Implen NanoPhotometer N50 ultramicro ultraviolet spectrophotometer (Thermo Scientific). The purity and integrity of RNA was determined in an Agilent 2100 Bioanalyzer. Finally, qualified total RNA was obtained, and the quality indicators were shown in Additional file: Table S4.

\section{cDNA library construction and sequencing}

Follow steps were required to build the library: purification and fragmentation of mRNA, synthesis and 


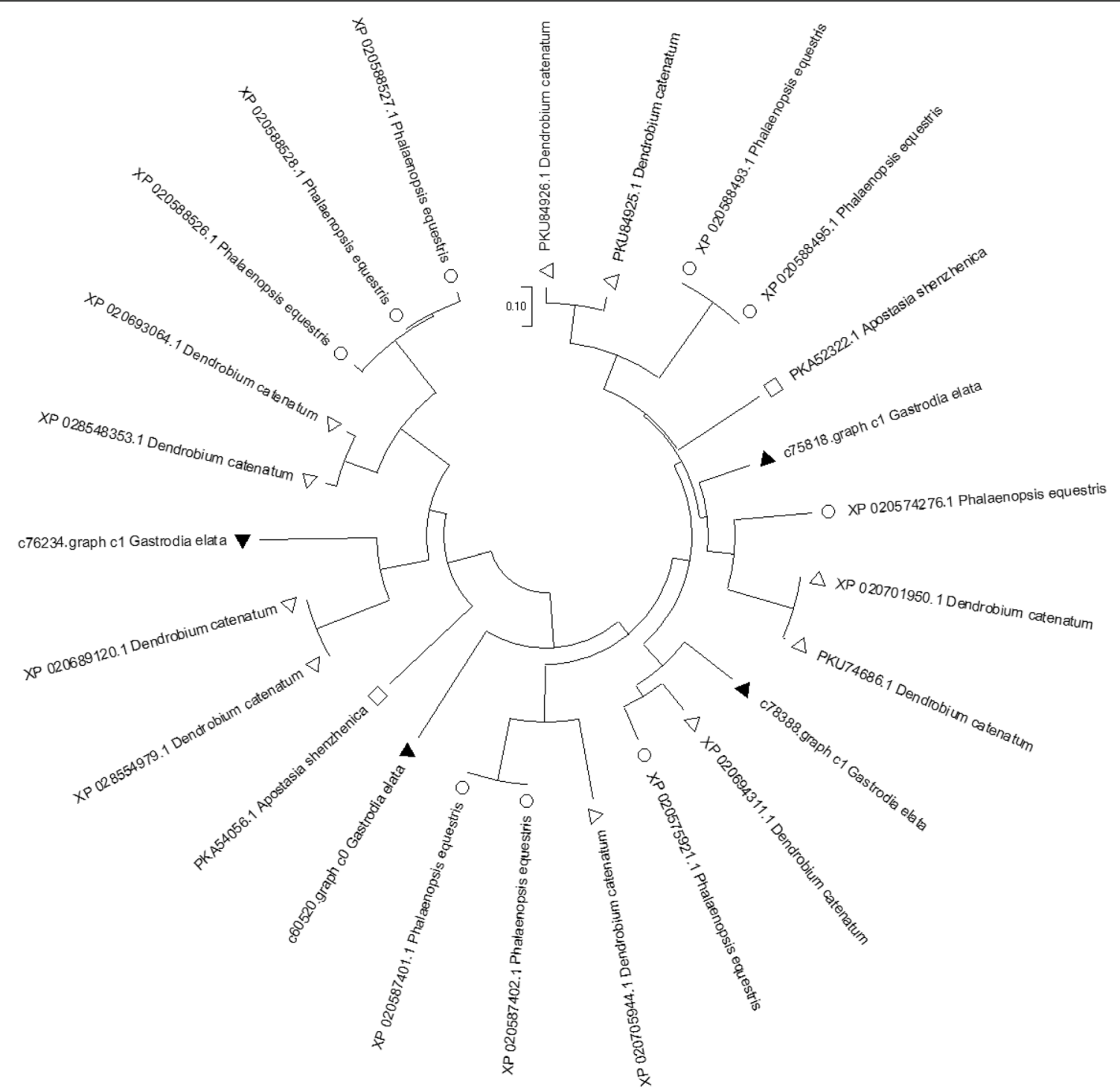

Fig. 10 Phylogenetic tree of TIFY10 in Orchidaceae plants. Branch length represents the credibility of homology. The shorter the branch, the higher the credibility of homology. Different species are displayed with different symbols. $\mathbf{\Delta}$ (solid triangle): G. elata Bl. f. glauca; $\Delta$ (hollow triangle): Dendrobium catenatum; o(circle): Phalaenopsis equestris; $\square($ (square): Apostasia shenzhenica

purification of double-stranded cDNA, the end repair or $\mathrm{dA}$ tail addition, junction ligation and USER (uracil-specific excision reagent) enzyme digestion, ligated products purification and fragments size classification, library amplification, magnetic bead purification or sorting of amplification products, library quality control [83]. cDNA library was checked for quality and quantity using Agilent 2100 Bioanalyzer. All RNA sequences of $150 \mathrm{bp}$ between $5^{\prime}$-terminal and 3 '-terminal was sequenced through Illumina Noveseq highflux sequencing platform [83]. Paired-end sequencing data was generated for each sample with $2 \times 150 \mathrm{bps}$ read lengths.

\section{Reads mapping and transcript de novo assembly}

The resulting reads called raw data were stored in fastq format. The raw data of each sequencing sample included two fastq files containing reads determined at both ends of all cDNA fragments. The quality of raw reads was assessed using the fastqc program (http:// www.bioinformatics.babraham.ac.uk/projects/fastqc/).

Data filtering on raw data to remove low quality reads and reads containing connector or poly- $\mathrm{N}$, we obtained high quality clean data.

Using Trinity software (https://github.com/trinityrnaseq/trinityrnaseq/wiki) with default parameters, the sequence assembly of clean data is carried out in 
combined assembly [84]. In this way, the sequencing depth can be increased indirectly, and transcripts with low expression abundance in G. elata Bl. f. glauca RNA samples can be assembled more completely. Clean data of each sample was aligned with assembled transcript or Unigene library to obtain mapped reads that matched transcript or Unigene library.

\section{Gene expression and annotation}

A Unigene supported by a minimum of three mapped high-quality reads was considered as expressed. This was done for the sake of reducing the false positive caused by independent statistical hypothesis test to a large number of gene expression values. FC (fold change) means the ratio of gene expression levels between healthy and diseased groups. Positive values show upregulation and negative values show down regulation of genes in diseased group. In addition, FPKM (reads per kilobase of exon model per million mapped reads) value is also a factor to be considered for DEGs identification. When gene expression abundance is small, that is to say, be with low signal values, it may not be detected in subsequent validation.

In organisms, different genes perform different biological functions, similar genes have similar functions. In order to predict the function of unknown genes and obtain their functional annotation information, all Unigenes were annotated into databases such as GO (Gene Ontology) [85], KEGG (Kyoto Encyclopedia of Genes and Genomes) [86], COG (clusters of orthologous groups) [87], KOG (clusters of euKaryotic Orthologous Groups) [88], eggNOG (Evolutionary Genealogy of Genes: Non-supervised Orthologous Groups) [89].

\section{qRT-PCR}

Using LightCycler 480 II real-time PCR system (Roche, Switzerland) and 2X SG Fast qPCR Master Mix (B639271, BBI, Canada), the expression levels of 9 genes in healthy and diseased G. elata Bl. f. glauca were relatively quantified. The primer sequences (Additional file: Table S5) were designed using Primer 5.0 and synthesized by Sangon Biotech (Shanghai) Co., Ltd. (https:// www.sangon.com). A two-step procedure (hold $95^{\circ} \mathrm{C} 3$ min; $45 \times\left(\right.$ duration $95^{\circ} \mathrm{C} 5 \mathrm{~s}$, anneal/extend $60^{\circ} \mathrm{C} 30 \mathrm{~s}$ )) was used for qRT-PCR assays. Each sample contained three biological replicates. The relative expression values were calculated with the $2^{-\Delta \Delta \mathrm{Ct}}$ method and normalized by the internal reference gene 18S rRNA (https://www. ncbi.nlm.nih.gov/nuccore/PVEL01000548[90, 91].

\section{Statistical analysis}

The data in this study was shown as the mean values of three biological duplication. Pearson correlation coefficient is used when discussing samples correlation [92] (Additional file: Table S6). DEGs were evaluated with the
DESeq2 package (http://www.bioconductor.org/packages/ release/bioc/html/DESeq.html). Benjamini-Hochberg method was used to correct the significant $p$ obtained from the original hypothesis test. The gene expression abundance was described by FPKM value. In addition, the differential expression and enrichment analysis were conducted using Fisher's exact test to obtain an adjusted $p$ with an FDR correction.

\section{Supplementary information}

Supplementary information accompanies this paper at https://doi.org/10. 1186/s12870-020-02653-4.

Additional file 1: Table S1. Sequencing and reads mapping.

Additional file 2: Table S2. GO terms enrichment of DEGs. KS: Kolmogorov-Smirnov test $(p<0.01)$.

Additional file 3: Table S3. Enriched KEGG pathways.

Additional file 4: Table S4. Concentration, purity and integrity of total RNA.

Additional file 5: Table S5. Primer pair sequences for qRT-PCR.

Additional file 6: Table S6. Correlation statistics between biological replicate samples. It is revealed with Pearson's correlation coefficient $r$. The closer $r^{2}$ is to 1, the stronger the correlation between two groups.

Additional file 7: Figure S1. (a) Base distribution and reads average rate of raw data. (b) Transcripts and Unigenes length distribution after de novo assembly.

Additional file 8: Figure S2. Monthly values accumulated from 1981 to 2010 in Jingyu County, Baishan City, Jilin Province, PR China.

\section{Abbreviations}

DEG: Differentially expressed Unigene; TF: Transcription factor; AD: Alzheimer's disease; PD: Parkinson's disease; BP: Biological process; CC: Cellular component; MF: Molecular function; KS: Kolmogorov-Smirnov test; nr: RefSeq non-redundant proteins; GO: Gene Ontology; KEGG: Kyoto Encyclopedia of Genes and Genomes; COG: Clusters of orthologous groups; KOG: Clusters of euKaryotic Orthologous Groups; eggNOG: Evolutionary Genealogy of Genes: Non-supervised Orthologous Groups; TR: Transcription regulator; PK: Protein kinases; CDPK: Calcium-dependent protein kinase; Rboh: Respiratory burst oxidase homolog; CNGC: Cyclic nucleotide gated channel; CML: Calmodulin-like protein; LRR: Leucine-rich repeat; MEKK1: Mitogen-activated protein kinase kinase kinase 1; MKK4/5: Mitogenactivated protein kinase kinase 4/5; RIN4: RPM1-interacting protein 4; HR: Hypersensitive response; GH3: Glycoside hydrolase 3; AHP: Histidinecontaining phosphotransfer protein; ARR-B: Two-component response regulator ARR-B family; PIF4: Phytochrome-interacting factor 4; ERF1: Ethylene-responsive transcription factor 1; JAZ: Jasmonate ZIM domaincontaining protein; AUX1: Auxin influx carrier; ARF: Auxin response factor; CRE: Cytokinin receptor enzyme; PP2C: Protein phosphatase 2C; EIN2: Ethylene-insensitive protein 2; BZR1/2: Brassinosteroid resistant 1/2; JAR1: Jasmonic acid-amino synthetase; COl1: Coronatine-insensitive protein 1; +p: Phosphorylation; -p: Dephosphorylation; +u: Ubiquitination; PTI: Pattern-triggered immunity; JA: Jasmonic acid; SA: Salicylic acid; ET: Ethylene; BR: Brassinosteroid; ETI: Effector-triggered immunity; SAR: Systemic acquired resistance; R genes: Resistance genes; HGe: Healthy tubers; DGe: Diseased tubers; FDR: False discovery rate; FC: Fold change

\section{Acknowledgements}

We are grateful to Zhaochun Li, the manager of JINGZHEN TIANMA Co., Ltd., for experimental materials. We extend sincere gratitude to Jingting Liu, Zhilong Zhang, Qiqi Mo, Jing Liang, Xue Zhang, Xue Wei, Yaqi Li, Peipei Gu, Mingyue Gao, Tao Lv, Fei Gao, Dehao Wang for material processing and technical assistance. We also thank BMKCloud (www.biocloud.net) for providing an analysis platform. 


\section{Authors' contributions}

YG contributed in research conceiving, material collection, and writing guidance. PZ provided technical support. YX assisted in experiment conducting. YW performed experiment and manuscript writing. All authors read and approved the final manuscript.

\section{Funding}

This work was supported by the Science and Technology Development Program of Jilin Province Fund (20190301079NY, 20170204017YY), National Key Research and Development Program Fund (2016YFC0500300). These funding bodies had no role in the design of the study and collection, analysis, and interpretation of data or in writing the manuscript.

\section{Availability of data and materials}

The sequence data generated during the current study are available in the NCBI SRA repository via accession numbers SAMN14380862 and SAMN14380861 (https://www.ncbi.nlm.nih.gov/bioproject/PRJNA612737). All data analyzed during this study are included in this published article and its additional files

\section{Ethics approval and consent to participate}

Not applicable.

\section{Consent for publication}

Not applicable.

\section{Competing interests}

The authors declared that they have no competing interests.

Received: 27 May 2020 Accepted: 15 September 2020

Published online: 29 September 2020

\section{References}

1. Chinese Pharmacopoeia Committee. Pharmacopoeia of the People's Republic of China. Beijing: China Medical Science and Technology Press; 2020. p. 59.

2. Lin YE, Chou ST, Lin SH, Lu KH, Panyod S, Lai YS, Ho CT, Sheen LY. Antidepressant-like effects of water extract of Gastrodia elata Blume on neurotrophic regulation in a chronic social defeat stress model. J Ethnopharmacol. 2018;215:132-9.

3. Liu Y, Gao J, Peng M, Meng H, Ma H, Cai P, Xu Y, Zhao Q, Si G. A review on central nervous system effects of gastrodin. Front Pharmacol. 2018;9:24.

4. Huang JY, Yuan YH, Yan JQ, Wang YN, Zhu CG, Guo QL, Shi JG, Chen NH. 20C, a bibenzyl compound isolated from Gastrodia elata, protects PC12 cells against rotenone-induced apoptosis via activation of the Nrf2/ARE/HO-1 signaling pathway. Acta Pharmacol Sin. 2016;37(6):731-40.

5. $\mathrm{Ng}$ CF, Ko CH, Koon CM, Xian JW, Leung PC, Fung KP, Ho Yin Edwin C, CBS $L$. The aqueous extract of rhizome of Gastrodia elata protected Drosophila and PC12 cells against beta-amyloid-induced neurotoxicity. Evid Based Complementray Altern Med. 2013;2013:516741.

6. Doo AR, Kim SN, Hahm DH, Yoo HH, Park JY, Lee H, Jeon S, Kim J, Park SU, Park HJ. Gastrodia elata Blume alleviates L-DOPA-induced dyskinesia by normalizing FosB and ERK activation in a 6-OHDA-lesioned Parkinson's disease mouse model. BMC Complement Altern Med. 2014;14:107.

7. Liu B, Gao JM, Li F, Gong QH, Shi JS. Gastrodin attenuates bilateral common carotid artery occlusion-induced cognitive deficits via regulating Aß-related proteins and reducing autophagy and apoptosis in rats. Front Pharmacol. 2018;9:405.

8. $\quad$ Ding YF, Bao XM, Lao LF, Ling YX, Wang QW, Xu SJ. P-Hydroxybenzyl alcohol prevents memory deficits by increasing neurotrophic factors and decreasing inflammatory factors in a mice model of Alzheimer's disease. J Alzheimers Dis. 2019;67(3):1007-19.

9. Chen PJ, Liang KC, Lin HC, Hsieh CL, Su KP, Hung MC, Sheen LY. Gastrodia elata Bl. Attenuated learning deficits induced by forced-swimming stress in the inhibitory avoidance task and Morris water maze. J Med Food. 2011 ; 14(6):610-7.

10. Jiang G, Hu Y, Liu L, Cai J, Peng C, Li Q. Gastrodin protects against MPP(+ )-induced oxidative stress by up regulates heme oxygenase-1 expression through p38 MAPK/Nrf2 pathway in human dopaminergic cells. Neurochem Int. 2014;75(9):79-88.
11. Zhang XL, Yuan YH, Shao QH, Wang ZZ, Zhu CG, Shi JG, Ma KL, Yan X, Chen $\mathrm{NH}$. DJ-1 regulating PI3K-Nrf2 signaling plays a significant role in bibenzyl compound 20C-mediated neuroprotection against rotenone-induced oxidative insult. Toxicol Lett. 2017;271(Complete):74-83.

12. Yuan Y, Jin X, Liu J, Zhao X, Zhou J, Wang X, Wang D, Lai C, Xu W, Huang J. The Gastrodia elata genome provides insights into plant adaptation to heterotrophy. Nat Commun. 2018;9(1):1615

13. Liu J, Zang P, Gao Y, Zhao Y, He Z, Zhu H, Wei X. First Report of Penicillium oxalicum Causing Blue Mould on Gastrodia elata BI. in Jilin Province, China. Plant Dis. 2019;103(9):2469.

14. Qiao M, Tian WG, Feng B, Yu ZF, Peng ZX. First report of soft rot associated with Ilyonectria robusta in Gastrodia elata. Plant Dis. 2019;103(10):2691.

15. Han M, Mi NC, Lee HR, Park EJ. First report of soft rot associated with Trichoderma hamatum in Gastrodia elata. Plant Dis. 2017;101(6):1048.

16. Jin Qiang Z, Xin T, Cheng Hong X, Jiao X, Qing Song Y, Xiao W, Da Hui L, Guang Wen Z, Fu Ming L, Wei Ke J, et al. Investigation, analysis and identification of disease of Gastrodia elata f. glauca. Zhongguo Zhong yao za zhi. 2020;45(3):478-84

17. Noshi M, Mori D, Tanabe N, Maruta T, Shigeoka S. Arabidopsis clade IV TGA transcription factors, TGA10 and TGA9, are involved in ROS-mediated responses to bacterial PAMP flg22. Plant Sci. 2016;252:12-21.

18. Chakraborty J, Sen S, Ghosh P, Jain A, Das S. Inhibition of multiple defense responsive pathways by CaWRKY70 transcription factor promotes susceptibility in chickpea under Fusarium oxysporum stress condition. BMC Plant Biol. 2020;20(1):319.

19. Cheng W, Jiang Y, Peng J, Guo J, Lin M, Jin C, Huang J, Tang W, Guan D, He $\mathrm{S}$. The transcriptional reprograming and functional identification of WRKY family members in pepper's response to Phytophthora capsici infection. BMC Plant Biol. 2020;20(1):256.

20. Dallery J, Zimmer M, Halder V, Suliman M, Pigné S, Le Goff G, Gianniou D, Trougakos I, Ouazzani J, Gasperini D, et al. Inhibition of jasmonate-mediated plant defences by the fungal metabolite higginsianin B. J Exp Bot. 2020; 71(10):2910-21.

21. Zang Z, Lv Y, Liu S, Yang W, Ci J, Ren X, Wang Z, Wu H, Ma W, Jiang L, et al. ZmERF105A novel ERF transcription factor, , Positively Regulates Maize Resistance to. Front Plant Sci. 2020;11:850.

22. Yao Z, Chen Q, Chen D, Zhan L, Zeng K, Gu A, Zhou J, Zhang Y, Zhu Y, Gao $W$, et al. The susceptibility of sea-island cotton recombinant inbred lines to Fusarium oxysporum $f$. sp. vasinfectum infection is characterized by altered expression of long noncoding RNAs. Sci Rep. 2019;9(1):2894.

23. Su X, Lu G, Guo H, Zhang K, Li X, Cheng H. The dynamic transcriptome and metabolomics profiling in Verticillium dahliae inoculated Arabidopsis thaliana. Sci Rep. 2018;8(1):15404

24. Gao Y, Wang W, Zhang T, Gong Z, Zhao H, Han GZ. Out of water: the origin and early diversification of plant R-genes. Plant Physiol. 2018;177(1):82-9.

25. Suzuki M, Wu S, Mimura M, Alseekh S, Mccarty DR. Construction and applications of a B vitamin genetic resource for investigation of vitamin dependent metabolism in maize. Plant J. 2019;101(2):442-54.

26. Song N, Ma L, Wang W, Sun H, Wang L, Baldwin IT, Wu J. An ERF2-like transcription factor regulates production of the defense sesquiterpene capsidiol upon Alternaria alternata infection. J Exp Bot. 2019;70(20):5895908.

27. Fitzgerald $\mathrm{H}$, Canlas $\mathrm{P}$, Chern M, Ronald $\mathrm{P}$. Alteration of TGA factor activity in rice results in enhanced tolerance to Xanthomonas oryzae pv. Oryzae. Plant J. 2005;43(3):335-47.

28. Hunter T. The age of crosstalk: phosphorylation, ubiquitination, and beyond. Mol Cell. 2007;28(5):730-8.

29. Ye J, Zhong T, Zhang D, Ma C, Wang L, Yao L, Zhang Q, Zhu M, Xu M. The auxin-regulated protein ZmAuxRP1 coordinates the balance between root growth and stalk rot disease resistance in maize. Mol Plant. 2019;12(3):360-73.

30. Wang L, Li Q, Liu Z, Surendra A, Pan Y, Li Y, Zaharia L, Ouellet T, Fobert P. Integrated transcriptome and hormone profiling highlight the role of multiple phytohormone pathways in wheat resistance against fusarium head blight. PLoS One. 2018;13(11):e0207036.

31. Wang S, Wang S, Sun Q, Yang L, Zhu Y, Yuan Y, Hua J. A role of cytokinin transporter in Arabidopsis immunity. Mol Plant-Microbe Interact. 2017;30(4): 325-33.

32. Wang J, Shi H, Zhou L, Peng C, Liu D, Zhou X, Wu W, Yin J, Qin H, Ma W, et al. OsBSK1-2, an orthologous of AtBSK1, is involved in rice immunity. Front Plant Sci. 2017:8:908 
33. Yuan M, Huang Y, Ge W, Jia Z, Song S, Zhang L, Huang Y. Involvement of jasmonic acid, ethylene and salicylic acid signaling pathways behind the systemic resistance induced by Trichoderma longibrachiatum $\mathrm{H} 9$ in cucumber. BMC Genomics. 2019;20(1):144.

34. Zhou S, Zhang YK, Kremling KA, Ding Y, Bennett JS, Bae JS, Kim DK, Ackerman $\mathrm{HH}$, Kolomiets MV, Schmelz EA, et al. Ethylene signaling regulates natural variation in the abundance of antifungal acetylated diferuloylsucroses and Fusarium graminearum resistance in maize seedling roots. New Phytol. 2019;221(4):2096-111.

35. Yuan P, Zhang C, Wang ZY, Zhu XF, Xuan YH. RAVL1 activates brassinosteroids and ethylene signaling to modulate response to sheath blight disease in rice. Phytopathology. 2018;108(9):1104-13.

36. Wang F, Wang C, Yan Y, Jia H, Guo X. Overexpression of cotton GhMPK11 decreases disease resistance through the gibberellin signaling pathway in transgenic Nicotiana benthamiana. Front Plant Sci. 2016;7:689.

37. Krattinger SG, Kang J, Bräunlich S, Boni R, Chauhan H, Selter LL, Robinson MD, Schmid MW, Wiederhold E, Hensel G, et al. Abscisic acid is a substrate of the $A B C$ transporter encoded by the durable wheat disease resistance gene Lr34. New Phytol. 2019;223(2):853-66.

38. Hatmi S, Villaume S, Trotel Aziz P, Barka EA, Clément C, Aziz A. Osmotic stress and $A B A$ affect immune response and susceptibility of grapevine berries to gray mold by priming polyamine accumulation. Front Plant Sci. 2018;9:1010.

39. Wang Q, Chen X, Chai X, Xue D, Zheng W, Shi Y, Wang A. The involvement of jasmonic acid, ethylene, and salicylic acid in the signaling pathway of -induced resistance to gray mold disease in tomato. Phytopathology. 2019;109(7):1102-14.

40. He Y, Zhang H, Sun Z, Li J, Hong G, Zhu Q, Zhou X, MacFarlane S, Yan F, Chen J. Jasmonic acid-mediated defense suppresses brassinosteroidmediated susceptibility to Rice black streaked dwarf virus infection in rice. New Phytol. 2017;214(1):388-99.

41. Xu L, Yang H, Ren L, Chen W, Liu L, Liu F, Zeng L, Yan R, Chen K, Fang X. Jasmonic acid-mediated aliphatic glucosinolate metabolism is involved in clubroot disease development in Brassica napus L. Front Plant Sci. 2018;9: 750.

42. Cai Y, Cai X, Wang Q, Wang P, Zhang Y, Cai C, Xu Y, Wang K, Zhou Z, Wang $C$, et al. Genome sequencing of the Australian wild diploid species Gossypium australe highlights disease resistance and delayed gland morphogenesis. Plant Biotechnol J. 2019;18(3):814-28.

43. Chen X, Wang H, Li X, Ma K, Zhan Y, Zeng F. Molecular cloning and functional analysis of 4-Coumarate:CoA ligase 4(4CL-like 1) from Fraxinus mandshurica and its role in abiotic stress tolerance and cell wall synthesis. BMC Plant Biol. 2019;19(1):231.

44. Wang L, Wang H, He S, Meng F, Zhang C, Fan S, Wu J, Zhang S, Xu P. GmSnRK1.1, a sucrose non-fermenting-1(SNF1)-related protein kinase, promotes soybean resistance to Phytophthora sojae. Front Plant Sci. 2019;10: 996.

45. Perochon A, Váry Z, Malla KB, Halford NG, Paul MJ, Doohan FM. The wheat SnRK1a family and its contribution to Fusarium toxin tolerance. Plant Sci. 2019;288:110217

46. Grimplet J, Agudelo Romero P, Teixeira RT, Martinez Zapater JM, Fortes AM. Structural and functional analysis of the GRAS gene family in grapevine indicates a role of GRAS proteins in the control of development and stress responses. Front Plant Sci. 2016;7:353.

47. Lim CW, Baek W, Lim S, Han SW, Lee SC. Expression and functional roles of the pepper pathogen-induced bZIP transcription factor CabZIP2 in enhanced disease resistance to bacterial pathogen infection. Mol PlantMicrobe Interact. 2015;28(7):825-33.

48. Shen L, Liu Z, Yang S, Yang T, Liang J, Wen J, Liu Y, Li J, Shi L, Tang Q, et al. Pepper CabZIP63 acts as a positive regulator during Ralstonia solanacearum or high temperature-high humidity challenge in a positive feedback loop with CaWRKY40. J Exp Bot. 2016;67(8):2439-51

49. Li X, Fan S, Hu W, Liu G, Wei Y, He C, Shi H. Two cassava Basic Leucine Zipper (bZIP) transcription factors (MebZIP3 and MebZIP5) confer disease resistance against cassava bacterial blight. Front Plant Sci. 2017:8:2110.

50. Zhao XY, Qi CH, Jiang H, Zhong MS, You CX, Li YY, Hao YJ. MdHIR4 transcription and translation levels associated with disease in apple are regulated by MdWRKY31. Plant Mol Biol. 2019;101(null):149-62.

51. Zhang F, Wang F, Yang S, Zhang Y, Xue H, Wang Y, Yan S, Wang Y, Zhang $Z$, Ma Y. MdWRKY100 encodes a group I WRKY transcription factor in Malus domestica that positively regulates resistance to Colletotrichum gloeosporioides infection. Plant Sci. 2019;286:68-77.
52. Kim JG, Mudgett MB. Tomato bHLH132 transcription factor controls growth and defense and is activated by effector XopD during pathogenesis. Mol Plant-Microbe Interact. 2019;32(12):1614-22.

53. Shan W, Chen JY, Kuang JF, Lu WJ. Banana fruit NAC transcription factor MaNAC5 cooperates with MaWRKYs to enhance the expression of pathogenesis-related genes against Colletotrichum musae. Mol Plant Pathol. 2016;17(3):330-8.

54. Liu Q, Yan S, Huang W, Yang J, Dong J, Zhang S, Zhao J, Yang T, Mao X, Zhu $X$, et al. NAC transcription factor ONAC066 positively regulates disease resistance by suppressing the $\mathrm{ABA}$ signaling pathway in rice. Plant $\mathrm{Mol}$ Biol. 2018;98(null):289-302

55. Meisrimler CN, Pelgrom AJE, Oud B, Out S, Van den AG. Multiple downy mildew effectors target the stress-related NAC transcription factor LsNAC069 in lettuce. Plant J. 2019;99(6):1098-115.

56. Shi H, Wang X, Ye T, Chen F, Deng J, Yang P, Zhang Y, Chan Z. The Cysteine2/Histidine2-type transcription factor ZINC FINGER OF ARABIDOPSIS THALIANA6 modulates biotic and abiotic stress responses by activating salicylic acid-related genes and C-REPEAT-BINDING FACTOR genes in Arabidopsis. Plant Physiol. 2014;165(3):1367-79.

57. Li W, Zhu Z, Chern M, Yin J, Yang C, Ran L, Cheng M, He M, Wang K, Wang $J$, et al. A natural allele of a transcription factor in rice confers broadspectrum blast resistance. Cell. 2017;170(1):114-126.e115.

58. Zhao Y, Chang X, Qi D, Dong L, Wang G, Fan S, Jiang L, Cheng Q, Chen X, Han D, et al. A novel soybean ERF transcription factor, GmERF113, increases resistance to Phytophthora sojae infection in soybean. Front Plant Sci. 2017; $8 \cdot 299$

59. Wei Y, Chang Y, Zeng H, Liu G, He C, Shi H. RAV transcription factors are essential for disease resistance against cassava bacterial blight via activation of melatonin biosynthesis genes. J Pineal Res. 2018;64(1):e12454.

60. Kim NY, Jang YJ, Park OK. AP2/ERF family transcription factors ORA59 and RAP2. 3 interact in the nucleus and function together in ethylene responses. Front Plant Sci. 2018;9:1675.

61. Zhang YL, Zhang CL, Wang GL, Wang YX, Qi CH, Zhao Q, You CX, Li YY, Hao YJ. The R2R3 MYB transcription factor MdMYB30 modulates plant resistance against pathogens by regulating cuticular wax biosynthesis. BMC Plant Biol. 2019;19(1):362.

62. Qiu Z, Yan S, Xia B, Jiang J, Yu B, Lei J, Chen C, Chen L, Yang Y, Wang Y, et al. The eggplant transcription factor MYB44 enhances resistance to bacterial wilt by activating the expression of spermidine synthase. J Exp Bot. 2019;70(19):5343-54

63. Pradhan SK, Pandit E, Nayak DK, Behera L, Mohapatra T. Genes, pathways and transcription factors involved in seedling stage chilling stress tolerance in indica rice through RNA-Seq analysis. BMC Plant Biol. 2019;19(1):352.

64. Xie Z, Lin W, Yu G, Cheng Q, Xu B, Huang B. Improved cold tolerance in switchgrass by a novel $\mathrm{CCCH}$-type zinc finger transcription factor gene, associated with ICE1-CBF-COR regulon and ABA-responsive genes. Biotechnol Biofuels. 2019:12(undefined):224.

65. Mithoe SC, Menke FL. Regulation of pattern recognition receptor signalling by phosphorylation and ubiquitination. Curr Opin Plant Biol. 2018;45(null): 162-70.

66. Liu F, Li X, Wang M, Wen J, Yi B, Shen J, Ma C, Fu T, Tu J. Interactions of WRKY15 and WRKY33 transcription factors and their roles in the resistance of oilseed rape to Sclerotinia infection. Plant Biotechnol J. 2018:16(4):911-25.

67. Hussain A, Li X, Weng Y, Liu Z, Ashraf M, Noman A, Yang S, Ifnan M, Qiu S, Yang $Y$, et al. Ralstonia CaWRKY22 acts as a positive regulator in pepper pesponse to by constituting networks with CaWRKY6, CaWRKY27, CaWRKY40, and CaWRKY58. Int J Mol Sci. 2018;19(5):1426.

68. Hussain RMF, Sheikh AH, Haider I, Quareshy M, Linthorst HJM. Arabidopsis WRKY50 and TGA transcription factors synergistically activate expression of PR1. Front Plant Sci. 2018:9:930.

69. Hui S, Hao M, Liu H, Xiao J, Li X, Yuan M, Wang S. The group I GH3 family genes encoding JA-lle synthetase act as positive regulator in the resistance of rice to Xanthomonas oryzae pv. Oryzae. Biochem Biophys Res Commun. 2019:508(4):1062-6.

70. Yang Y, Ahammed G, Wan C, Liu H, Chen R, Zhou Y. Comprehensive analysis of TIFY transcription factors and their expression profiles under jasmonic acid and abiotic stresses in watermelon. Int J Genomics. 2019; 2019(undefined):6813086.

71. Ebel C, BenFeki A, Hanin M, Solano R, Chini A. Characterization of wheat (Triticum aestivum) TIFY family and role of Triticum Durum TdTIFY11a in salt stress tolerance. PLoS One. 2018;13(7):e0200566. 
72. Chini A, Ben-Romdhane W, Hassairi A, Aboul-Soud MA. Identification of TIFY/JAZ family genes in Solanum lycopersicum and their regulation in response to abiotic stresses. PLoS One. 2017;12(6):e0177381.

73. Oblessuc P, Obulareddy N, DeMott L, Matiolli C, Thompson B, Melotto M. JAZ4 is involved in plant defense, growth, and development in Arabidopsis. Plant J. 2019;101(2):371-83.

74. Lee S, Rojas C, Oh S, Kang M, Choudhury S, Lee H, Allen R, Pandey S, Mysore K. Nucleolar GTP-binding protein 1-2 (NOG1-2) interacts with jasmonate-ZIMDomain protein 9 (JAZ9) to regulate stomatal aperture during plant immunity. Int J Mol Sci. 2018;19(7):1922.

75. Liu S, Zhang P, Li C, Xia G. The moss jasmonate ZIM-domain protein PnJAZ1 confers salinity tolerance via crosstalk with the abscisic acid signalling pathway. Plant Sci. 2019;280(undefined):1-11.

76. Ma Q, Zhou Q, Chen C, Cui Q, Zhao Y, Wang K, Arkorful E, Chen X, Sun K, Li $X$. Isolation and expression analysis of CsCML genes in response to abiotic stresses in the tea plant (Camellia sinensis). Sci Rep. 2019;9(1):8211.

77. Wu X, Qiao Z, Liu H, Acharya BR, Li C, Zhang W. CML20, an Arabidopsis calmodulin-like protein, negatively regulates guard cell ABA signaling and drought stress tolerance. Front Plant Sci. 2017;8:824.

78. An J, Zhang X, Bi S, You C, Wang X, Hao Y. The ERF transcription factor MdERF38 promotes drought stress-induced anthocyanin biosynthesis in apple. Plant J. 2019;101(3):573-89.

79. Midhat U, Ting M, Teresinski H, Snedden W. The calmodulin-like protein, CML39, is involved in regulating seed development, germination, and fruit development in Arabidopsis. Plant Mol Biol. 2018;96(null):375-92.

80. Sravankumar T, Akash NN, Kumar R. A ripening-induced SIGH3-2 gene regulates fruit ripening via adjusting auxin-ethylene levels in tomato (Solanum lycopersicum L.). Plant Mol Biol. 2018;98(null):455-69.

81. Henry E, Yadeta K, Coaker G. Recognition of bacterial plant pathogens: local, systemic and transgenerational immunity. New Phytol. 2013;199(4):908-15.

82. Zhan H, Zhou H, Sui Y, Du X, Wang W, Dai L, Sui F, Huo H, Jiang T. The rhizome of Gastrodia elata Blume - An ethnopharmacological review. J Ethnopharmacol. 2016;189:361-85.

83. Podnar J, Deiderick H, Huerta G, Hunicke-Smith S. Next-generation sequencing RNA-Seq library construction. Curr Protoc Mole Biol. 2014;106:4.21.21-19.

84. Grabherr M, Haas B, Yassour M, Levin J, Thompson D, Amit I, Adiconis X, Fan L, Raychowdhury R, Zeng Q, et al. Full-length transcriptome assembly from RNASeq data without a reference genome. Nat Biotechnol. 2011;29(7):644-52.

85. Ashburner M, Ball C, Blake J, Botstein D, Butler H, Cherry J, Davis A, Dolinski K, Dwight S, Eppig J, et al. Gene ontology: tool for the unification of biology. The gene ontology consortium. Nat Genet. 2000;25(1):25-9.

86. Kanehisa M, Goto S, Kawashima S, Okuno Y, Hattori M. The KEGG resource for deciphering the genome. Nucleic Acids Res. 2004;32:D277-80.

87. Tatusov R, Galperin M, Natale D, Koonin E. The COG database: a tool for genome-scale analysis of protein functions and evolution. Nucleic Acids Res. 2000;28(1):33-6.

88. Koonin E, Fedorova N, Jackson J, Jacobs A, Krylov D, Makarova K, Mazumder R, Mekhedov S, Nikolskaya A, Rao B, et al. A comprehensive evolutionary classification of proteins encoded in complete eukaryotic genomes. Genome Biol. 2004:5(2):R7.

89. Huerta-Cepas J, Szklarczyk D, Forslund K, Cook H, Heller D, Walter M, Rattei T, Mende D, Sunagawa S, Kuhn M, et al. eggNOG 4.5: a hierarchical orthology framework with improved functional annotations for eukaryotic, prokaryotic and viral sequences. Nucleic Acids Res. 2016;44:D286-93.

90. Wan X, Zou L, Zheng B, Wang Y. Circadian Regulation of Alternative Splicing of Drought-Associated CIPK Genes in Dendrobium catenatum (Orchidaceae). Int J Mol Sci. 2019;20(3): 688

91. Livak KJ, Schmittgen TD. Analysis of relative gene expression data using real-time quantitative PCR and the 2(-Delta Delta C(T)) method. Methods. 2001;25(4):402-8.

92. Anders $\mathrm{S}$, Huber W. Differential expression analysis for sequence count data. Genome Biol. 2010;11(10):R106.

\section{Publisher's Note}

Springer Nature remains neutral with regard to jurisdictional claims in published maps and institutional affiliations.

\section{Ready to submit your research? Choose BMC and benefit from:}

- fast, convenient online submission

- thorough peer review by experienced researchers in your field

- rapid publication on acceptance

- support for research data, including large and complex data types

- gold Open Access which fosters wider collaboration and increased citations

- maximum visibility for your research: over $100 \mathrm{M}$ website views per year

At BMC, research is always in progress.

Learn more biomedcentral.com/submissions 